\title{
Herencia social y logros educativos en Argentina ¿Meritocracia o herencia social?
}

\section{Social background and educational achievement in Argentina. Meritocracy or social background?}

\author{
María Sol TORRES MINOLDO y Marcos Javier ANDRADA \\ Universidad de Granada - CONICET-UNLaR (Argentina)
}

Recibido: Septiembre 2012

Aceptado: Enero 2013

\section{Resumen}

Este artículo discute la legitimidad de aquellas políticas públicas cuyo impacto distributivo reproduce desigualdades económicas ya presentes en la sociedad. Para ello se intenta rebatir la presunción de que éstas sean resultado de mecanismos meritocráticos, que asignan compensaciones y castigos en función del esfuerzo o la virtud.

Así, con el objetivo de constatar la escasa relevancia de la meritocracia para explicar la estratificación socioeconómica en sociedades desiguales, y vislumbrar si en cambio la desigualdad de oportunidades tiene un rol relevante en su etiología, se abordará la relación entre logros educativos y origen socioeconómico, en base al estudio del caso argentino.

Palabas clave: Desigualdad Social, desarrollo económico, éxito escolar, herencia social, clase social

\begin{abstract}
This article discusses the legitimacy of those public policies which replicates distributional impact economic inequalities already present in society. It tries to rebut the presumption that these mechanisms are the result of meritocratic rewards and punishments they assign a function of effort or virtue.

Thus, in order to verify the negligible importance of meritocracy to explain socioeconomic stratification in unequal societies, and glimpse if you use the inequality of opportunity has a significant role in its etiology, will address the relationship between educational achievement and socioeconomic background, based on the study of the Argentine case.
\end{abstract}

Keywords: social inequality, economic environment, school success, social background, social structure 
La seguridad social pública incide sobre la distribución del ingreso, tanto a partir de sus maneras de financiamiento, como de distribución. En algunos casos su impacto es redistributivo (progresiva o regresivamente), y en otros tiende a reproducir, o reforzar, las desigualdades distributivas existentes en la sociedad.

Este último caso es naturalizado muchas veces como un criterio "justo", tanto por funcionarios y políticos, como por la sociedad en su conjunto ${ }^{1}$.

El consentimiento de un esquema de prestaciones diferenciales, vinculadas a las diferencias de ingresos durante la vida activa, se asocia a una implícita legitimación de las desigualdades de ingresos entre los miembros de la sociedad, propia de ideologías liberales o corporativistas. Discutir las premisas de la misma constituye el objetivo del presente artículo.

La existencia de un criterio de justicia meritocrático, según el cual el mérito y el esfuerzo deben ser premiados, supone que lo justo será precisamente que exista desigualdad. Sin embargo, en este trabajo se cuestionará la verosimilitud de la meritocracia ${ }^{2}$ como explicación de la estratificación social. El objetivo es, por tanto, constatar la escasa relevancia del mecanismo meritocrático para explicar la estratificación socioeconómica en sociedades desiguales, en base al estudio del caso argentino.

Si se reconociera que la verdadera causa de la estratificación no es el mérito y esfuerzo individual sino la desigualdad de oportunidades, caerá por tierra la legitimación de la desigualdad en nuestra sociedad, y por tanto, también de la asignación, en la práctica de la protección social, de prestaciones que reproducen la desigualdad en base a un "reemplazo" del nivel de ingresos durante la vida activa.

${ }^{1}$ En el caso de las jubilaciones, por ejemplo, tanto el discurso liberal como el corporativista derivan en el respaldo a sistemas de tipo contributivo, en los cuales los futuros beneficiarios participan en la financiación del sistema y los beneficios se relacionan con esa historia de aportes: ya sea de manera actuarial en el esquema liberal, o como prestaciones definidas por una "tasa de reemplazo" en el modelo corporativista. En suma, el monto de las prestaciones está en ambos casos vinculado a los ingresos durante la vida activa. Podría pensarse que el fundamento de las prestaciones desiguales se halla legitimado casi exclusivamente por la práctica misma de la financiación contributiva. Sin embargo, la existencia de la misma opera como un refuerzo simbólico a la idea de la prestación como una "retribución" al aporte económico hecho al sistema, pero no como su fundamento último ya que: aun cuando el financiamiento contributivo pierde terreno, la propuesta de prestaciones igualitarias parece encontrarse fuera de debate.

${ }^{2}$ La meritocracia requiere que gente con idénticos niveles de esfuerzo y de preferencias gocen de idénticos resultados. Cualquier desigualdad en los resultados debe poder ser explicada perfectamente con las diferencias de esfuerzos y preferencias (Paes De Barros, Ferreira, Molinas Vega, \& Saavedra Chanduvi, 2008). 
Así, al constatar la incidencia de mecanismos sociales estructurales en la asignación diferencial de oportunidades de vida, e impugnar la presunta legitimidad de las diferencias de ingreso, será posible argumentar en favor del principio de equidad colectiva como principio directriz de la protección social, es decir, de la (re)redistribución progresiva al diseñar modalidades de financiamiento y estructuras de prestaciones en el sistema de seguridad social.

\section{La legitimación de la estratificación socioeconómica en los discursos liberal y corporativista}

De acuerdo con Esping Andersen (1993) todos los regímenes de bienestar producen estratificación social ${ }^{3}$. Los estados de bienestar abrazan diferentes principios redistributivos, algunos de los cuales pueden promover una mayor igualdad de resultados o de oportunidades, mientras que otros pueden de hecho trabajar en la dirección opuesta ${ }^{4}$ (Esping Andersen \& Myles, 2007, pág. 2).

La idea de justicia detrás de un sistema de protección social que reproduce deliberadamente los desiguales logros en el mercado laboral, se asociaría a la legitimidad que habría en el origen de aquella estratificación que reproduce. Lograr mayor salario, mayor ahorro y una inserción más segura en el mercado de trabajo sería presuntamente producto del mérito: para el modelo liberal, del esfuerzo y el ingenio; para el corporativista, de la virtud y el trabajo. De este modo las desventajas socioeconómicas no son pensadas como responsabilidad social porque la estratificación social es el resultado de responsabilidades o virtudes individuales o, en todo caso, familiares.

El sistema de protección social de los regímenes liberales, siguiendo la clasificación de Esping Andersen (1993), se caracteriza por que la protección social a gran escala es concebida bajo la lógica del seguro social, en que los beneficios se definen en función del aporte hecho al sistema por el beneficiario, suponiendo una relación actuarial entre contribuciones hechas al sistema y prestaciones recibidas. Es que se considera que el individuo ahorrativo, emprendedor y trabajador debe ser recompensado y que el mercado es el más eficiente sistema para asignar los recursos en función de ese criterio. De este modo, el seguro social, con el objeto de respetar la distribución meritocrática, debe imitar la lógica del mercado para lograr cumplir eficientemente su objetivo.

\footnotetext{
${ }^{3}$ La ubicación de las personas en estratos o grupos jerárquicamente organizados según su acceso a recursos [sean éstos económicos, sociales o políticos] es lo que desde la sociología llamamos estratificación (Martínez Franzoni, 2005a, pág. 5).

4 "Welfare states embrace distinct redistributive principles, some of which may promote more equality of outcomes or of opportunities, while others may actually work in the opposite direction” [traducción propia].
} 
Por su parte, los modelos de protección social de tipo laboralista o, en términos de Esping Andersen, conservadores o corporativistas, pueden parecer a primera vista muy distantes del modelo liberal. En ellos el papel del Estado es fundamental, y la protección social tiene como objetivo instrumentar derechos. Sin embargo, se trata de derechos vinculados al status y la clase social, cuyo acceso y monto dependen de la trayectoria laboral. Si bien el beneficiario ya no es el individuo, los son estratos sociales segmentados por status o clase. De este modo, el sistema corporativista imita también el esquema distributivo que ya está establecido en la sociedad, promoviendo prestaciones y formas de financiamiento que refuerzan las tendencias configuradas en el mercado. "El énfasis del Estado en elevar las diferencias de status significa que su impacto redistributivo [vertical] es insignificante” (Esping Andersen, 1993).

Como se desprende de ambos casos, de cómo entendamos que se produce la pobreza o el acceso a un mercado de trabajo informal o mal pago, depende el que avalemos un sistema basado en los mismos criterios distributivos, o por el contrario, se busque modificar dicha distribución.

Cuando una parte de la desigualdad de resultados (como el ingreso, los salarios, los avances ocupacionales, o los logros educativos) puede ser atribuida a circunstancias, esto refleja desigualdad de oportunidades en una sociedad (Paes De Barros, Ferreira, Molinas Vega, \& Saavedra Chanduvi, 2008, pág. 63). Si la desigualdad de oportunidades es la explicación más certera de la estratificación social, no será difícil encontrar consenso social en cuanto a que la misma es injusta y, por lo tanto, ilegítima.

\section{Herencia social: movilidad social y transmisión intergeneracional de la pobreza}

La herencia social consiste en un condicionamiento que actúa cuando las oportunidades de vida de las personas son restringidas o favorecidas por su origen socioeconómico. Cuando la herencia social es determinante en la situación socioeconómica que los miembros de la sociedad logran alcanzar, no es posible responsabilizar individualmente a los ciudadanos por ellas.

La existencia o no de herencia social dependerá, por supuesto, de la sociedad a la que uno se refiera, el protagonismo del mercado en la estratificación, el rol del Estado en las oportunidades de los ciudadanos, el funcionamiento de la economía, etc. Sin embargo es un rasgo común a las economías capitalistas en general, especialmente en el actual mundo globalizado, la desigualdad social que se genera en función de fuerzas estructurales que se sobreponen al individuo. Incluso en países donde los ingresos económicos generales son altos, donde los niveles de desocupación y/o de informalidad son modestos, y donde el Estado ha logrado universalizar el acceso a la educación básica, la estratificación social sigue teniendo más que ver con diferencias heredadas socialmente que con el resultado de comportamientos individuales. Esta diferencia de 
oportunidades asignada socialmente sería más fuerte en países con mayor desigualdad estructural. $^{5}$

En una sociedad con efectiva igualdad de oportunidades, y donde sólo las virtudes individuales y los esfuerzos particulares definen los logros socioeconómicos, deberían encontrarse en cada estrato social, personas de múltiples orígenes, sin que predomine ninguna tendencia. La razón es que si se asume que las virtudes, aptitudes y méritos no se transmiten genéticamente, no se podría explicar fácilmente el hallazgo de que aquellos que nacen en un cierto estrato tengan mayores probabilidades de alcanzar determinados logros.

Por eso, si un alto porcentaje de ciudadanos no modifican la situación socioeconómica de su hogar de origen, y se constata una alta correlación entre el origen social y los logros alcanzados en la vida adulta, se estará frente a un fuerte indicio de la existencia en la sociedad de desigualdad de oportunidades que condicionan los logros de los individuos, quienes contarán con dificultades y facilidades en función no de sus méritos, sino del azar de nacer en una u otra familia.

\section{El caso argentino}

El caso argentino es paradigmático en la cuestión que aquí nos ocupa: sistemas de protección social contributivos que reproducen la desigualdad; altos niveles de desigualdad social; y significativo peso de las legitimaciones meritocráticas de dichas desigualdades. Allí, las legitimaciones de tipo liberal y corporativo son parte del consenso generalizado al momento de referirse a la protección social, apoyar una determinada estructura distributiva de asignaciones (prestaciones) o nuevos impuestos. Por su parte, el sistema de seguridad social es principalmente de tipo contributivo, combinado con prestaciones focalizadas de tipo liberal y, de manera muy residual, cuenta con asignaciones no contributivas que ciertamente son objeto de polémicas frecuentes en torno a su legitimidad.

Con todas estas características, el caso argentino resulta ejemplar para analizar la etiología de la estratificación y determinar si la misma es consecuencia principalmente de comportamientos, méritos y virtudes individuales, o en realidad operan fuerzas sociales más allá del control del sujeto.

\footnotetext{
${ }^{5}$ Los estudios comparativos a nivel internacional dejan bastante claro que las posibilidades que la gente tiene para salir de una situación de pobreza están relacionadas con las desigualdades a nivel global: sociedades con grandes desigualdades sociales, como EE.UU., tienen muchas más probabilidades de generar situaciones duraderas de pobreza que sociedades más igualitarias (Bradbury et al. 2001, citado en Esping-Andersen, 2006).
} 


\section{Limitaciones metodológicas}

Constatar la existencia de la herencia social o medir los niveles de movilidad social impone la necesidad de contar con datos que son difíciles y costosos de generar.

En la mayoría de los estudios se observan alguno o algunos de los siguientes problemas: falta de homogeneidad de la muestra, una sola observación de los ingresos obtenida de encuestas contemporáneas, encuestas retrospectivas para obtener datos sobre los padres (que en la mayoría de los casos conllevan respuestas poco confiables debido al tiempo transcurrido y/o al desconocimiento de la información requerida), muestras pequeñas y falta de variables de control que permitan chequear la información recabada (Dearden et al. 1997, citado en Jiménez, 2011).

La definición misma de pobreza, o estrato socioeconómico de origen, suele ser uno de los problemas fundamentales en las metodologías utilizadas para estudios de herencia social en Argentina.

Aldas Carrol y Morán (2001) desarrollaron una metodología que goza de cierto reconocimiento en el ambiente académico. En ella, con el objeto de determinar en qué grado la pobreza se transmite de padres a hijos, se analiza la evolución de los logros educacionales entre la generación de los jefes de hogar y de los hijos. En este abordaje se restringe el análisis a los jóvenes de 18 a 25 años que conviven con sus padres, en hogares definidos como "pobres". Estos autores caracterizan un hogar como pobre cuando el jefe cuenta con un nivel de instrucción primaria incompleta como máximo.

"Esta operativización se propone debido a la falta de otra información sobre el pasado familiar de los encuestados que permitan categorizar de manera satisfactoria su hogar de procedencia como pobre o no pobre por otros criterios, como los ingresos o las necesidades básicas insatisfechas” (Golovanevsky, 2007, pág. 388).

Finalmente se asume que alcanzar el nivel medio completo sería una forma de salir de la trampa de la pobreza. La elección de la culminación del ciclo de enseñanza secundaria como probabilidad a explicar responde a que, este umbral educativo, permite la obtención de un ingreso que otorga cierta probabilidad de situarse fuera de la pobreza en América Latina (Aldaz Carroll \& Morán, 2001). Sin embargo, se requerirían, al menos 12 años de educación para lograr una nivel de competencias asociado a una alta inserción laboral ${ }^{6}$. De acuerdo a información, se requerirían entre 10 y 12 años de educación para disminuir la probabilidad de ser pobre (Infante. 2000, citado en Guadalupe, 2002).

\footnotetext{
${ }^{6}$ Alfabetismo funcional en siete países de América Latina, UNESCO/OREALC, Santiago, 2000. 
Con estas categorías y las bases de la ECV-2001, diversos autores (Golovanevsky, 2007; Ferrari Recalde, 2008) proceden a estudiar el grado de herencia social en nuestro país.

Los autores que recurren al uso de esta metodología reconocen que la misma alberga diversas limitaciones por la falta de datos de panel. Por ejemplo, los criterios para definir como pobres a padres e hijos se aplican en función de observarlos en edades muy diferentes, mucho más tempranas en los hijos. Esto se debe a que al hacerse mayores e irse del hogar paterno, se pierde la relación entre padres e hijos en las encuestas de hogares.

Otras limitaciones que pueden objetarse a esta metodología tienen que ver con la definición misma de las categorías de hogar pobre y no pobre. El considerar sólo los logros educativos deja de lado un importante factor de transferencia de ingresos y propiedad: la herencia material. Así, una persona que hereda tierras, propiedades, empresas o cualquier factor productivo generador de ingresos, así como recursos materiales concretos, no podrá ser considerado pobre y claramente tendrá la posibilidad de invertir en la educación de sus hijos. Este es el caso del devenir de algunos negocios familiares que luego de formar un capital considerable se pueden constituir en una herencia que modificará las oportunidades de las próximas generaciones. En estos casos, la salida de la pobreza de las mismas que, según las categorías utilizadas en esta metodología, sería un caso de superación de la herencia social, en realidad es una constatación del mismo.

Por otro lado, es cuestionable en sí mismo que el status socioeconómico se defina a partir de los logros educativos. Si bien es cierto que pueden existir fuertes correlaciones entre los mismos y las oportunidades laborales, la educación secundaria completa no garantiza necesariamente la oportunidad de salir de la pobreza, y cada día su importancia se disminuye frente al aumento de las exigencias del mercado de trabajo formal de mayores capacitaciones laborales para acceder a trabajos en mejores condiciones, y mejor pagados. Otra vez, los resultados pueden tender a subestimar el impacto de la herencia social. Por otro lado, al relacionar logros educativos del entorno familiar con los logros del joven, las correlaciones encontradas pueden hablar de reproducción cultural más que de condicionamiento socioeconómico. Es necesario tener presente que la relación entre logros educativos y logros socioeconómicos no es automática, aun cuando el supuesto es que sí lo sea en sociedades meritocráticas, no puede darse por sentado que efectivamente así ocurra.

Otro abordaje metodológico relevante es el del Observatorio Social (2001), que examina la movilidad intergeneracional de la estructura social argentina en función de la permanencia o cambio en términos de inserción social que ha experimentado la población con respecto a la posición social del grupo familiar de origen. Se comparan el estrato socio-ocupacional del principal sostén del hogar cuando el entrevistado tenía 14 años con la inserción socio-ocupacional del entrevistado en un tiempo reciente. La 
población objeto de estudio son personas de entre 18 y 69 años, con ocupación u oficio laboral.

El problema es que la información del estrato socio-ocupacional de origen se basa en un criterio poco fiable, ya que se define en función de la percepción del sujeto sobre su pasado, a veces lejano, y en función de criterios disímiles para definir en cada caso ese estrato. Una vez más, la falta de datos de panel constituye una limitación clave que pone en duda los resultados del análisis empírico realizado por estas investigaciones.

\section{Un abordaje más realista: la herencia social y los logros educativos}

Dada la inexistencia de datos de panel, es poco factible asociar nivel socioeconómico de una persona que ha constituido su propio hogar, con el de su origen, dada la dificultad de determinar su origen socioeconómico de manera retrospectiva. Es por eso que en este artículo se intenta determinar la incidencia del nivel socioeconómico en las oportunidades de vida en una instancia previa a la constitución de un nuevo hogar y el consecuente alcance de determinado nivel socioeconómico. De este modo, se relaciona el origen socioeconómico con los logros educativos, algo que puede ser medido en simultáneo cuando el individuo aún vive en el hogar de sus padres y no es jefe de familia. En ese caso es posible determinar el nivel socioeconómico de su hogar, que es a su vez su hogar de origen, y observar sus logros educativos.

Aunque los logros educativos no sean en absoluto un sinónimo necesario de futuros logros socioeconómicos ${ }^{7}$, es útil su observación a los fines de determinar la existencia o no de una distribución igualitaria de oportunidades y, por lo tanto, de un funcionamiento efectivo de la meritocracia en la sociedad.

De este modo, la discusión sobre la responsabilidad individual o colectiva de los logros socioeconómicos de los miembros de la sociedad, se aborda con el cuestionamiento de uno de los principales supuestos de la interpretación meritocrática de la estratificación: que los logros educativos sean un indicador del mérito.

Generalmente se parte de la premisa de que son los logros educativos aquellos que generan un acceso a los mejores puestos laborales, y así los logros socioeconómicos se distribuyen por el mérito ${ }^{8}$. Pero si, aunque se aceptara esta

${ }^{7}$ La consideración de la educación como una inversión a futuro ha sido un tema ampliamente desarrollado por los economistas teóricos del Capital Humano, desde mediados del siglo XX, sin embargo, este enfoque al no considerar los rendimientos no pecuniarios y las externalidades derivadas de la inversión en la educación deja una parte importante de los efectos de la educación sin considerar.

${ }^{8}$ Incluso este supuesto puede ser puesto en duda. Los estudios de Golthorpe relativizan la importancia de la calificación en los logros socioeconómicos, mostrando que hay otras 
última presunción, se constatara que los logros educativos no son una medida exclusivamente del esfuerzo, la verosimilitud del funcionamiento de la meritocracia estaría en principio rebatida.

\section{Aproximación empírica/estadística. Antecedentes de estudios empíricos en Argentina}

La asociación constatada en este país, entre logros educativos y variables socioeconómicas ha sido reconocida ya en diversos estudios que han abordado dicha relación en diferentes momentos y regiones del mismo. Todos ellos han dejando constancia de que, en algún modo, la desigualdad educativa se relaciona con la desigualdad socioeconómica.

Un estudio reciente encontró que en los principales centros urbanos del país la población de 14 a 18 años que no asiste a la escuela y no tiene secundaria completa es del 36,4\% en hogares del primer quintil, mientras que es de tan sólo el 3,7\% del quintil más elevado (SIEMPRO, 2001). López (2002) mostró también que en los principales conglomerados urbanos, un tercio de los niños de 14 años pertenecientes a hogares del primer quintil terminan fuera de la escuela cuando llegan a los 17 años, mientras que menos del 5\% de los que conforman hogares del quinto quintil enfrentan esa situación. Independientemente de los grupos de edad examinados, o si se examina asistencia u logros educativos, los jóvenes desertores están sobre-representados en los hogares pertenecientes a los quintiles de ingresos más bajos (Binstock \& Cerrutti, 2005).

Por su parte, varios organismos internacionales se refieren a la falta de equidad en los países de América Latina en cuanto al acceso al conocimiento de los alumnos, tanto de Educación Básica como Media o Secundaria (UNESCO, 2000a y 200b, UNESCO/ OCDE, 2003, IEA, 1999, en Blanco y Cusato, 2005).

La autora Susana Torrado (1994) analiza también la relación entre logros educativos y origen socioeconómico. Pero la caracterización socioeconómica no tiene que ver con los logros educativos del jefe sino con su trabajo e ingresos. Así, Torrado se refiere a los estratos marginales o carenciados, obreros y de clase media. Por otro lado, los logros educativos en sí mismos no son interpretados como sinónimo de pertenencia a uno u otro estrato social. Según Torrado, estos diferentes logros educativos pueden repercutir más o menos en los logros socioeconómicos de acuerdo al contexto social, particularmente a los niveles generales de movilidad estructural. La influencia específica de estos comportamientos con respecto a las posibilidades de ascenso social dependerá de la cantidad y calidad de la movilidad social que se experimente en cada situación concreta. En contextos de intensa movilidad estructural ascendente, los factores demográficos pueden quizá dificultar la promoción social pero

variables que llevan a los empleadores a contratar, y que son de carácter adscriptivo, es decir, que no tienen que ver con el mérito (Goldthorpe \& Jackson). 
no impedirla. En tanto que, en situaciones de movilidad estructural neutra o descendente, los factores demográficos pueden efectivamente convertirse en un escollo insalvable para el ascenso (Torrado, 1994).

Respecto a lo metodológico, la autora recurre a datos censales de 1980 y 1991, de manera que su trabajo tiene menos limitaciones, pero solo puede restringirse a mediciones hechas cada 10 años. Por otro lado, tampoco cuenta con datos de panel que le permitan hacer un seguimiento del mismo caso en dos mediciones diferentes.

Las principales observaciones del estudio de Susana Torrado han sido, para 1980:

- $\quad$ El acceso de los niños al nivel primario, aunque desigual (87\% contra 92\%) no establece diferencias sustanciales entre estratos polares.

- Se constata que la finalización del nivel primario introduce notorias discriminaciones interestratos: el porcentaje de adultos sin primaria completa entre los marginales (60\%) representa una vez y media al de los estratos obreros y tres veces el de la clase media.

- El retraso escolar es altamente diferencial: el porcentaje de niños que cursan el nivel primario estando retrasados respecto de la edad normal fluctúa alrededor de $20 \%$ en la clase media, sube a cerca de $35 \%$ entre los obreros y de nuevo a $42-47 \%$ entre los marginales.

- El acceso al nivel secundario, por el contrario, sí introduce de entrada una neta diferenciación social: en la clase media, la tasa de escolarización oscila entre $55-60 \%$, alcanza 33\% entre los obreros (es decir, aproximadamente la mitad de los valores modales dentro de la clase media), y disminuye otra vez a la mitad (16\%) en el caso de los carenciados.

- Por último, la escolarización en el nivel superior, si bien agranda mucho las distancias entre los estratos, los deja en análogas posiciones relativas, siendo notable el hecho de que tanto la clase obrera como los estratos carenciados están virtualmente excluidos de este nivel educativo.

Los datos de 1991 confirman estas tendencias, poniendo mejor de manifiesto la profundidad de la fractura que separa a los "pobres" de los "no-pobres" en materia de educación:

- La matrícula primaria es 10 puntos inferior entre los primeros que entre los segundos.

- El índice de retraso escolar no ya duplica sino triplica el de los unos con respecto al de los otros.

- Además, otras dimensiones del acceso diferencial a la educación son ahora discernibles: se constata que el analfabetismo es un fenómeno casi privativo de los pobres. 
Según la autora, altos niveles de movilidad estructural de una sociedad disminuyen la influencia de estos factores en la determinación de la desigualdad. Según constata en sus estudios, la intervención del Estado mediante políticas públicas es lo que puede incrementar la movilidad estructural y reducir el peso de la herencia social. Por tanto, es lícito deducir, cuanto más liberales las sociedades, mayor será el peso de la herencia social.

\section{Un aporte a la constatación de desigualdad de oportunidades como trasfondo de la desigualdad educativa}

Con el fin de proporcionar un análisis actualizado de la relación entre logros educativos y origen socioeconómico en Argentina, se realizó el análisis estadístico de las bases de la Encuesta Permanente de Hogares (EPH) correspondientes al último trimestre del año 2011.

Como se mencionó precedentemente, existe una gran dificultad para evaluar las variables nivel educativo y origen socioeconómico sin datos de panel. Por ello, definir el origen socioeconómico de los individuos considerados en la muestra fue el principal desafío metodológico. En primer término, se rechaza recurrir al nivel educativo de los padres como sinónimo del mismo, por razones de interpretación teórica. Se recortó la muestra en función de aquellos jóvenes que aún forman parte de su hogar paterno, es decir, de origen, y se ha tomado en consideración la situación económica del hogar en función de su ingreso y la composición del mismo.

De este modo, hemos seleccionado una muestra de jóvenes de entre 18 y 25 años que aún habitan su hogar paterno en condición de hijos (excluyéndolos si cumplían la condición de jefes de hogar), asumiendo que la situación socioeconómica del hogar en el momento de la medición corresponde a una situación de largo plazo, es decir que los ingresos del hogar lo posicionan distributivamente de manera igual o similar a los años de formación educativa del joven. Este supuesto es válido en tanto se supone que la situación económica del hogar es estable ya que en los años previos los padres ya estaban insertados en el mercado de trabajo y la jefatura del hogar no ha cambiado.

Para evitar definiciones de pobreza que pueden ser controvertidas, dada la escasa credibilidad de los índices de precios actuales ${ }^{9}$ y evitar divisiones en clases cuya fundamentación sea difícil de ofrecer, se dividirá la población en quintiles de ingresos, con el objeto de observar la asociación de los ingresos y los logros educativos. La consideración de la condición distributiva antes que de pertenencia a una u otra clase, o

${ }^{9}$ La estimación de la canasta básica, que en definitiva fija los límites de la indigencia y ponderada los límites de la pobreza en Argentina, es un tema muy controvertido, desde que se cuestionará las estadísticas públicas a partir del año 2007. La inflación informada por el INDEC, oficialmente ha sido seriamente cuestionada incluso por ex empleados de la institución y por consultoras privadas. 
bien de la condición de pobreza, permitirá apreciar la influencia de la desigualdad, y no solo de las carencias por sí mismas. De este modo hemos medido el origen socioeconómico por medio de la variable Condición distributiva. Igualmente, en una sociedad como la argentina, con altos niveles de desigualdad, se puede suponer que los quintiles extremos corresponden a casos de pobreza y riqueza.

Considerando entonces el ingreso del hogar, y ponderándolo en relación a los individuos que lo constituyen, hemos obtenido una medida de ingreso que permite comparar la situación económica de los individuos de la muestra ${ }^{10}$.

En cuanto a la variable nivel educativo, se consideró el máximo nivel educativo alcanzado, y si fue o no completado.

Dado que se trabaja con la población mayor de 18 años, se estipula que a esa edad ya deberían haberse completado los estudios secundarios e iniciados los superiores. Por eso, en los casos de estudios primarios o secundarios incompletos, se asume que la probabilidad de finalizar esos estudios en el futuro será baja. En el único caso que los estudios incompletos pueden deberse a la edad es en los universitarios. Pero una población aun mayor en la muestra hubiera dificultado reconocer el origen socioeconómico.

También es de suponer que en algunos casos existen probabilidades de finalizar los estudios secundarios, pero se trata claramente de estudiantes con retraso escolar por lo que su consideración en función de su origen socioeconómico sigue siendo relevante.

En el gráfico se vera en cada quintil, es decir, en cada estrato distributivo, los logros educativos alcanzados.

10 Para considerar su situación distributiva no se procede a dividir simplemente el ingreso del hogar por el número de individuos que lo constituyen, sino por el número de unidades personas del hogar. Así el nivel de ingreso de los diferentes hogares es comparable más allá de la diferencia en el número, la edad y el género de sus integrantes Por ello, los quintiles de ingreso aquí presentados son los ingresos totales familiares, divididos no exactamente en el número de individuos del hogar sino en unidades equivalente de individuo, según consumo calórico que se fija por la edad y el sexo. Para determinar las unidades personas del hogar se consideran los valores recomendados por los nutricionistas respecto a la cantidades mínimas de calorías y otros nutrientes (V.g.: proteínas, hierro, vitaminas) que requieren las personas según edad y género. Así se atribuye un valor a cada integrante del hogar en función de su edad y género, pudiendo determinar un número final de unidades personas en el hogar. (INDEC, 2003) 


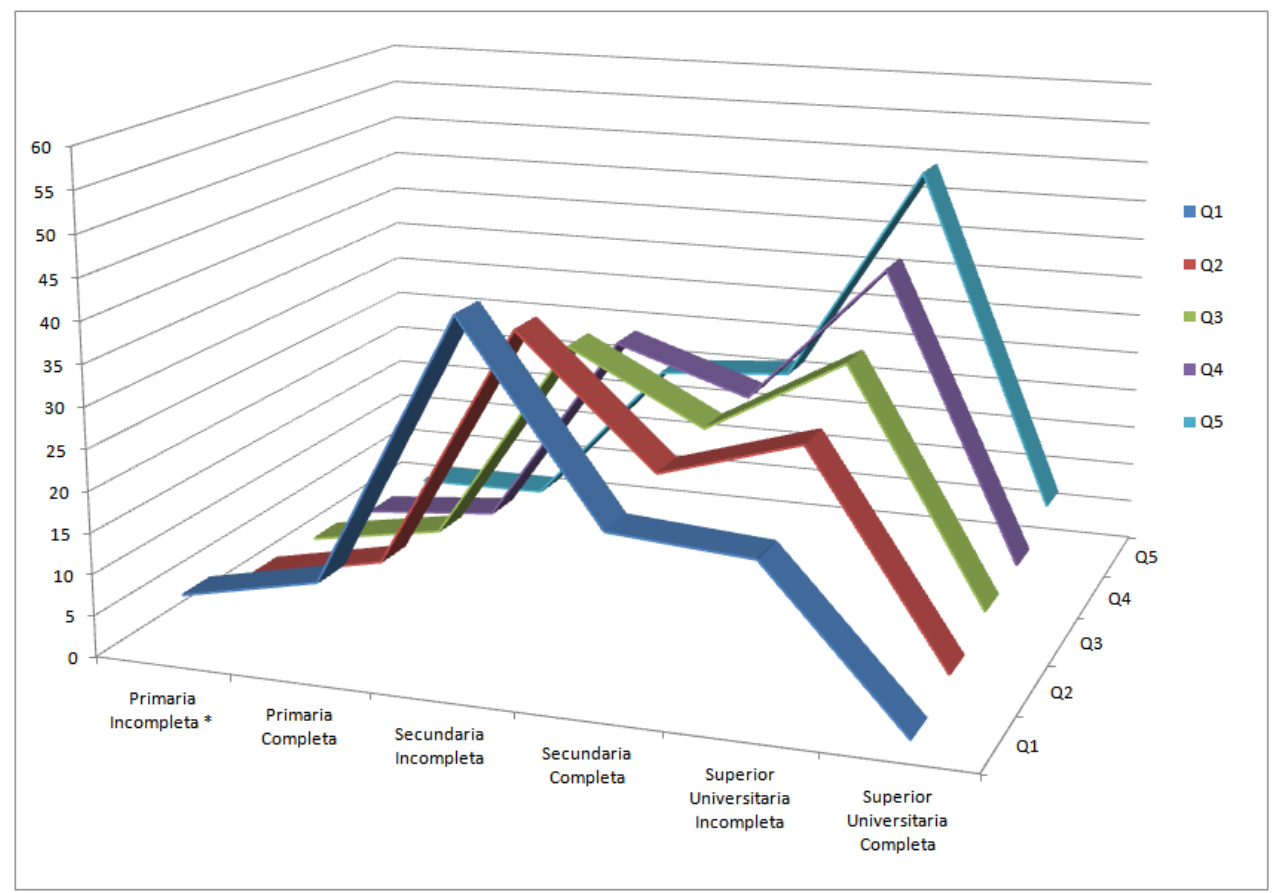

Gráfico 1. Distribución según nivel de estudio alcanzado y quintil de ingreso declarado. Ambos Sexos. Argentina. Año 2011

Fuente: Elaboración propia a partir de datos del Instituto Nacional de Estadísticas y Censos (INDEC). Encuesta Permanente de Hogares (EPH) 2011 (4to Trimestre).

* Incluye educación especial y personas sin instrucción

Los resultados muestran una tendencia muy evidente en la correspondencia entre logros educativos y nivel socioeconómico.

Así, en los quintiles de ingresos más bajos predominan logros educativos modestos o nulos, mientras que, por el contrario, a medida que mejoran los ingresos del hogar, se accede a estudios de mayor nivel.

La comparación de los valores extremos de la distribución socioeconómica de la muestra permite observar la expresión más cruda de la desigualdad de logros educativos: la mayoría de los jóvenes de menores ingresos no ha completado siquiera los estudios secundarios. En tanto, la mayoría de los jóvenes más aventajados en la distribución del ingreso ha accedido e incluso logrado completar la formación universitaria.

Sin embargo, la relación entre logros educativos y origen socioeconómico es evidente a lo largo de todos los niveles distributivos, pudiéndose reconocer una 
relación directamente proporcional casi constante entre estas dos variables: a menores ingresos, menores logros educativos, y viceversa.

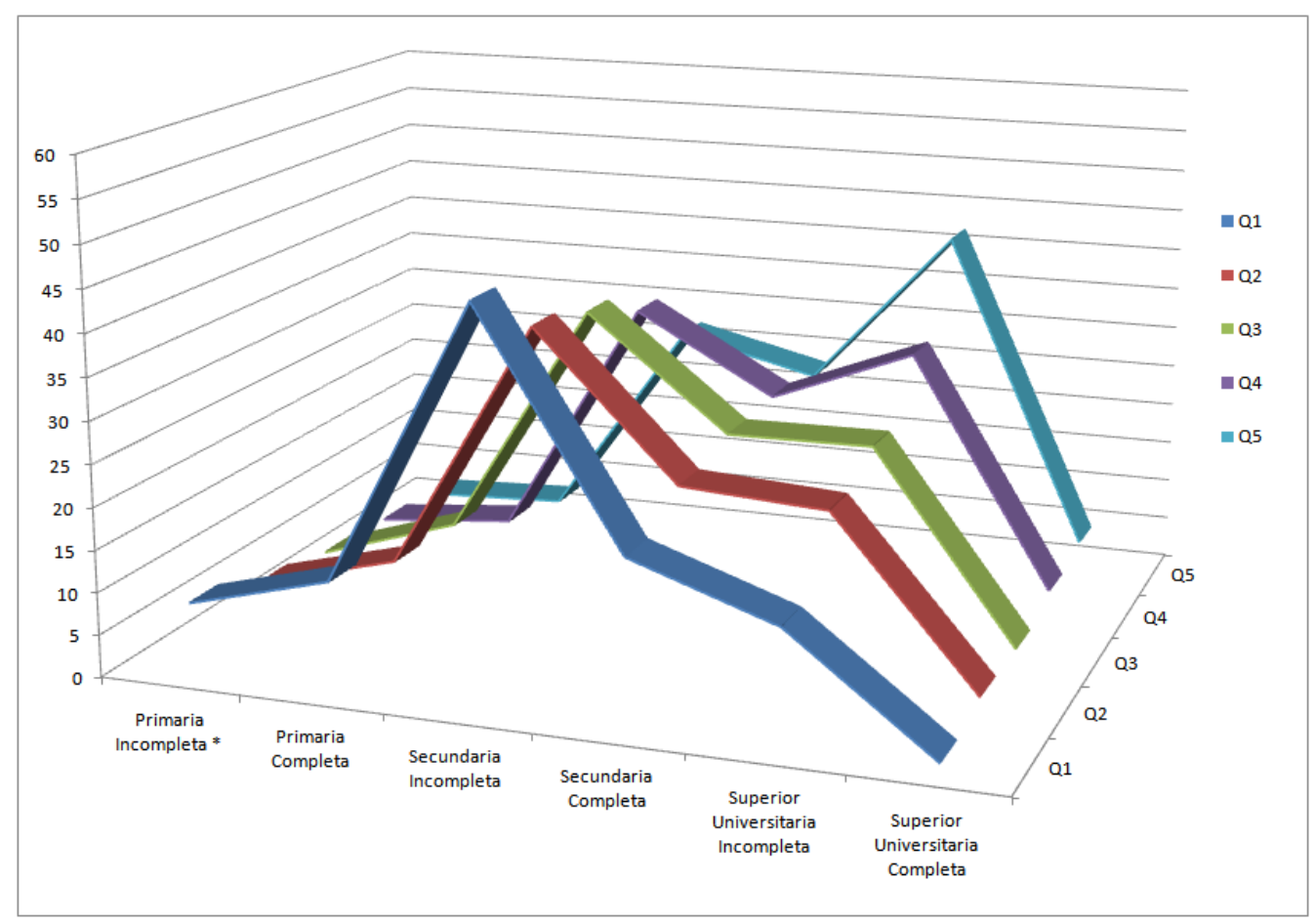

Gráfico 2. Distribución según nivel de estudio alcanzado y quintil de ingreso declarado. Hombres. Argentina. Año 2011

Fuente: Elaboración propia a partir de datos del Instituto Nacional de Estadísticas y Censos (INDEC). Encuesta Permanente de Hogares (EPH) 2011 (4to Trimestre).

* Incluye educación especial y personas sin instrucción

Al dividir la muestra por género y observar en cada uno esta relación entre logros educativos y origen socioeconómico, se encuentran algunas diferencias a la relación general, con mayores logros educativos por parte de las mujeres de los quintiles más altos, y una mayor restricción de logros educativos en el caso de los hombres de los estratos más bajos. Estas diferencias probablemente se expliquen porque los hombres se lanzan al mercado antes y eso les da a las mujeres mayor probabilidad de permanecer en el sistema. 


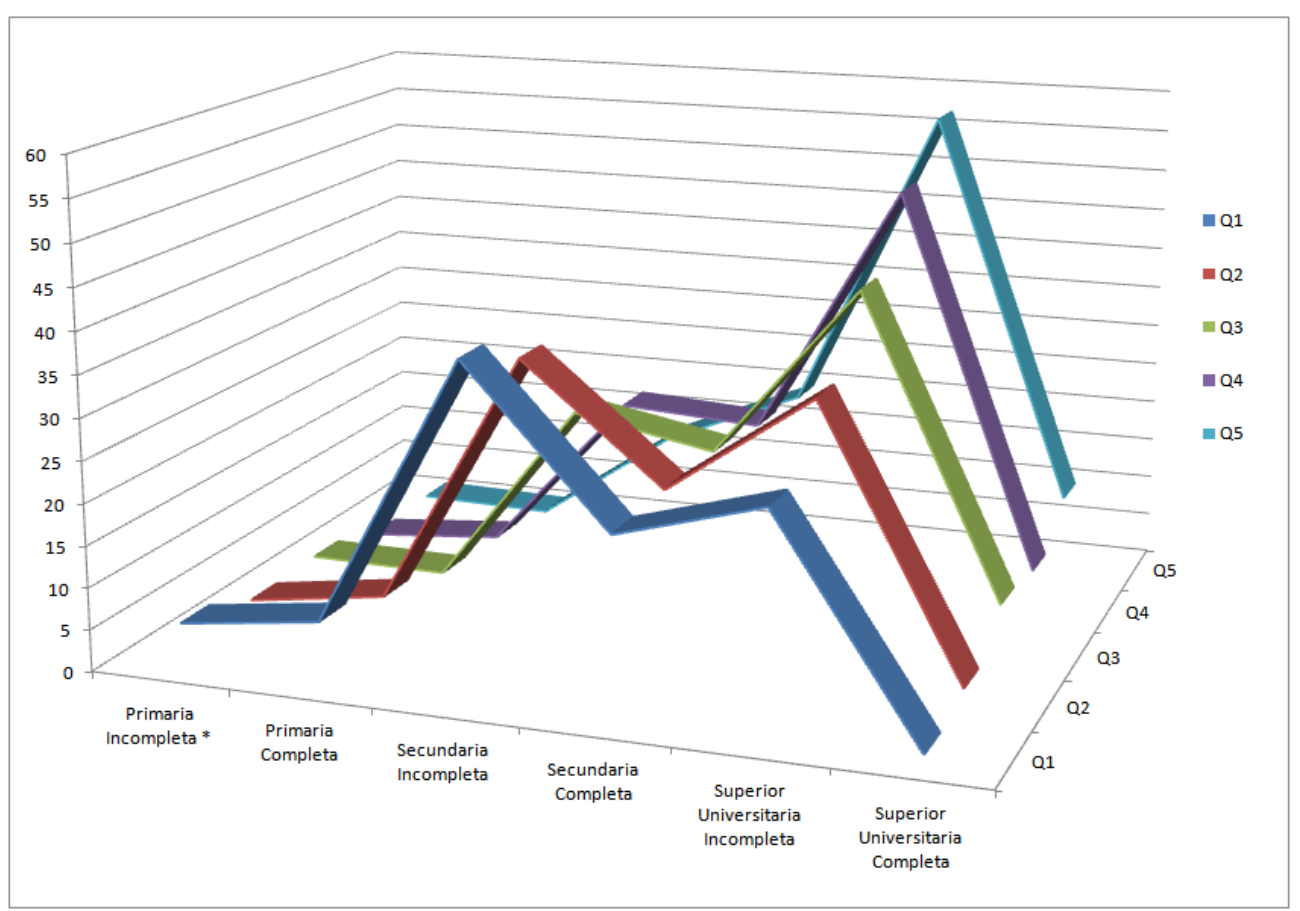

Gráfico 3. Distribución según nivel de estudio alcanzado y quintil de ingreso declarado. Mujeres.

Argentina. Año 2011

Fuente: Elaboración propia a partir de datos del Instituto Nacional de Estadísticas y Censos (INDEC). Encuesta Permanente de Hogares (EPH) 2011 (4to Trimestre).

* Incluye educación especial y personas sin instrucción

\section{La meritocracia como obstáculo de la meritocracia}

Luego de la exposición de los resultados del análisis estadístico, es posible sostener que los logros educativos, el indicador por excelencia del mérito, no debe sus diferencias sólo, ni principalmente, a los méritos individuales. Es que en gran medida están condicionados por la influencia de factores adscriptivos, de modo que "los niños y niñas de orígenes menos privilegiados tienen que mostrar mayor mérito que aquellos procedentes de clases más favorecidas para alcanzar posiciones de clase similares” (Méndez \& Gayo, 2007).

Aún en sociedades donde el Estado proporciona universalmente la educación primaria, e incluso secundaria, como es el caso de la Argentina, nacer y criarse en una familia pobre o rica suponen oportunidades claramente diferentes en el largo plazo. Los logros educativos, el tiempo de la vida dedicado a la educación, las capacitaciones 
a las que se accede, los activos sociales dados por las relaciones sociales del grupo de pertenencia; es decir, las principales condiciones que pesan en el mercado para definir los logros socioeconómicos de una persona, son condicionadas, restringidas o facilitadas a cada uno, de acuerdo al origen social de la familia en la que nació y creció.

"Harrington (1962), señala que la explicación real de por qué los pobres están donde están es haber cometido el error de nacer de los padres equivocados, en el sector equivocado de la ciudad, o en el grupo racial o étnico equivocado. Una vez cometido este error inicial, pueden ser ejemplo de voluntad y moralidad, pero esto no cambiará su realidad, que es la de sus remotas posibilidades de salir de ese mundo de pobreza" (Golovanevsky, 2007, pág. 382).

Esta situación, ¿`se debe a una aplicación imperfecta del sistema de asignación meritocrática de premios y castigos? De hecho, la causa que hace a la meritocracia devenir en pura retórica, aparece como consecuencia inevitable de la meritocracia misma. Al generarse desigualdades sociales como resultado de la atribución de castigos y penalizaciones, lógicamente el resultado será la desigualdad social. Cuando ésta afecte las oportunidades de vida de las próximas generaciones, mediante el impacto de la herencia social, ya no podrán atribuirse los diferentes logros socioeconómicos a méritos, virtudes y responsabilidades individuales exclusivamente (ni tan siquiera principalmente).

De este modo, la desigualdad de oportunidades resulta una consecuencia necesaria de cualquier sistema que genere estratificación, aunque la misma se deba al mérito, siempre que no haya límites ni compensaciones a la estratificación que produce. Si se asume que la desigualdad en sí misma, con o sin justificación, opera como productora de nuevas desigualdades, se deduce que estas últimas serán, con toda certeza, ilegítimas y determinadas por la desigualdad de oportunidades. Así, en la medida que haya adultos pobres, aunque supusiéramos que esa pobreza es su propia responsabilidad, sus hijos crecerán en la pobreza y sus oportunidades estarán marcadas por esa realidad. Viceversa, aun si los más adinerados han cosechado virtuosamente su fortuna, sus hijos crecerán en medio de ventajas y facilidades ajenas a sus cualidades o virtudes particulares. De este modo, la existencia de desigualdad, más allá de cuál sea su origen, es generadora de desigualdades ilegítimas.

"Estaríamos en presencia de una estructura de oportunidades sesgada en favor de quienes ya están en posesión de un activo social (...) Una estructura de oportunidades caracterizada por un proceso de acentuación de la discriminación del tipo que Robert Merton identificó como "el efecto Mateo". Este efecto se refiere a la acumulación de ventajas y desventajas, en una determinada estructura social. (...) La acumulación diferencial de las ventajas opera de tal manera que, parafraseando a los 
evangelistas Mateo, Marcos y Lucas, 'al que tiene, se le dará más, y tendrá de sobra; pero al que no tiene, hasta lo poco que tiene se le quitará”"” ${ }^{11}$ (Atria, 2004, págs. 38-40).

Algunos autores liberales discuten la existencia del efecto mateo considerando que, si bien se puede reconocer algún tipo de incidencia del origen social, la determinación es relativa. La incidencia dependería, presuntamente, de los méritos de los individuos en lo que respecta a su capacidad para sobreponerse a las dificultades que "la vida" le imponga. Pero dentro de esta consigna de aliento al trabajo se oculta el reconocimiento de que no existe igualdad de oportunidades. Y no queda claro cómo se justificaría el hecho de que para un mismo logro se exija mucho más esfuerzo a un individuo que a otro. La conclusión de estos razonamientos suele culminar en que en todos los casos depende de uno su destino: en el caso extremo, el pobre que nació desfavorecido es responsable por no haberse sobrepuesto a su suerte. Este razonamiento es una trampa lógica orientada responsabilizar a los pobres de su pobreza. Cuando arbitrariamente las exigencias son diferentes, claramente los logros no constituyen la compensación equitativa de las virtudes y méritos de cada individuo.

Un argumento que refuerza al anterior es la utilización ejemplificadora de los casos minoritarios de movilidad social. V. G., cuando personas de clases bajas logran salir de la pobreza son señaladas como la prueba de que, con esfuerzo y voluntad, sobre todo con trabajo, salir de la pobreza es posible. Se trataría simplemente de una elección. Una vez más el razonamiento es incorrecto: El que algunos salgan de la pobreza no es contradictorio con la existencia de diferencias de oportunidades y ventajas adquiridas de acuerdo con el origen socioeconómico, por lo tanto, tampoco prueba nada que contradiga esta aseveración. Menos oportunidades y desventajas no suponen necesariamente imposibilidad para la movilidad ascendente. Del mismo modo que ventajas y facilidades para los de clase alta no garantizan evitar el descenso socioeconómico. Pero cuando influye la herencia social, el distanciamiento del origen social no es lo que predomina, y no tiene el mismo costo para todos los individuos. Las posibilidades de ganar y perder no son las mismas, los esfuerzos demandados no son los mismos, y por tanto los resultados no premian o penalizan iguales esfuerzos o carencias, sino que existe la mediación de la clase social, ya sea como una protección extra o, por el contrario, como una situación de vulnerabilidad.

Muchos defensores del ideal meritocrático desde una teoría liberal, consideran que la igualdad de resultados no es deseable, sino que sólo lo es la de oportunidades. Sin embargo, como respaldan los argumentos e investigaciones hasta aquí expuestos, la igualdad de oportunidades es inconcebible en sociedades con altísimos niveles de desigualdad socioeconómica, especialmente, la que afecta a niños y jóvenes durante sus etapas de formación. Es por ello que algunos autores han enfatizado un conjunto de factores institucionales o macro sociales que pueden facilitar o dificultar los logros

${ }^{11}$ San Mateo, 25, 29 
educacionales de las personas. Entre ellos, un primer énfasis, ha sido puesto en la necesidad que tienen las diferentes sociedades de alcanzar niveles mínimos de igualdad económica y cultural para lograr una mayor igualdad de oportunidades educacionales. En este sentido, la expansión de las políticas sociales de bienestar parece haber contribuido a una mayor igualdad de oportunidades educacionales en países como Suecia o los Países Bajos (De Graff \& Ganzeboom, 1993). En definitiva, es claro que las desigualdades en los logros educacionales son difíciles de eliminar y que ellas tienen efectos en la reproducción de las desigualdades intra e intergeneracionales (Torche \& Wormald, 2004).

\section{Conclusiones}

La pauta meritocrática constituye un principio de justicia tan arraigado que resulta útil como base para establecer consensos sociales. Sin tomar distancia de la ética del trabajo y de la valoración del mérito como criterio de asignación diferencial de los recursos sociales, es posible introducir la responsabilidad colectiva a la comprensión de la desigualdad y, luego, el principio de equidad colectiva como un criterio distributivo justo.

En una sociedad meritocrática lo justo sería que los resultados socioeconómicos de las personas estén principalmente determinados por su esfuerzo particular, es decir, que cada uno obtenga en relación a sus méritos. Sin embargo, a la luz de las constataciones empíricas, esta no parece ser la principal explicación de la estratificación social en nuestra sociedad.

En Argentina, los hallazgos empíricos son consistentes con la afirmación de que la suerte de nacer en un hogar con más o menos recursos será determinante en las oportunidades de alcanzar mayores logros educativos. En consecuencia, aun concediendo que realmente fueran los logros educativos los que determinan el nivel de ingresos y el tipo de trabajo al que se accede, el origen social será en gran medida responsable de los logros económicos de cada individuo en tanto la desigualdad de oportunidad impacta en el proceso educativo mismo.

Dado que el capitalismo se maneja con asignaciones diferenciales (teóricamente basadas en premio y castigo al esfuerzo), está en su lógica producir estratificación. Como evidencian los hechos, en una sociedad de realidades materiales muy disímiles la igualdad de oportunidades es poco realista. Es por ello que, cuanto más desigual es una sociedad (generalmente asociado a mayor libertad del mercado para distribuir y menos políticas sociales compensatorias), mayor es la herencia social, como constatan estudios que refieren una asociación negativa entre los niveles de desigualdad de una sociedad y su apertura, indicada por los índices de movilidad intergeneracional.

La impugnación de la pretendida legitimidad de la estratificación producida por el mercado proporciona fundamento a la adopción del criterio de equidad colectiva: Si la estratificación que resulta del mercado es injusta, es fundamental asumir socialmente la 
responsabilidad de interferir sobre dicho esquema y compensar de ese modo a quienes son víctimas de injusticias de una sociedad en la que las probabilidades de obtener el bienestar son diferentes para las personas, más allá de sus aptitudes y esfuerzos.

En consideración a esto, el Estado puede y debe utilizar criterios distributivos contrarios a las desigualdades arbitrarias e injustas que la sociedad produce de manera permanente. Si la desigualdad tiende a producir desigualdad de oportunidades, los criterios de intervención del Estado deben constituir un contrapeso a la estratificación propia del mercado, y no el refuerzo de la misma.

\section{Referencias bibliográficas}

ALDAZ CARROLL, E., \& MORÁN, R. (2001). Escaping the poverty trap in Latin America : the role of family factors. Cuadernos de Economía , 38(114), 155-190.

AÑÓN, M. J., \& MIRAVET, P. (2005). Paradojas del familiarismo en el Estado del bienestar: mujeres y renta básica. Cuadernos de Relaciones Laborales, 23(2), 101121.

ATRIA, R. (Octubre de 2004). Estructura ocupacional, estructura social y clases sociales. Serie Política Sociales(96).

BINSTOCK, G., \& CERRUTTI, M. (2005). Carreras Truncadas: El abandono escolar en el nivel medio en la Argentina. Buenos Aires, Argentina: UNICEF.

BLANCO, S., \& CUSATO, R. (2005). Desigualdades educativas en América Latina: todos somos responsables. Santiago de Chile: UNESCO.

CEPAL. (1994). Panorama Social de América Latina. (N. Unidas, Ed.) Santiago de Chile: CEPAL.

CEPAL. (1997). La estructura del empleo en los últimos 15 años. En Panorama social de América Latina (págs. 57-92). Santiago de Chile, Chile: CEPAL.

CEPAL. (2000). Equidad, desarrollo y ciudadanía. Santiago de Chile, Chile: Comisión Económica para América Latina y el Caribe.

CEPAL. (2007). Calidad de la educación: las desigualdades más allá del acceso y la progresión educativa. Panorama social de América Latina, 157-200.

CEPAL. (2010). La educación frente a la reproducción intergeneracional de la desigualdad y la exclusión: situación y desafíos en América Latina. Panorama social de América Latina, 85-136.

DE GRAFF, P. M., \& GANZEBOOM, H. B. (1993). Family background and educational attainment in the Netherlands for the 1891-1960 birth cohorts. En Y. Shavit, \& H. P. Blossfeld (Edits.), Persistent Inequality: Changing Educational Attainment in iThirteen Countries (págs. 75-99). Boulder: Westview Press. 
ESPING ANDERSEN, G. (1993). Los tres mundos del Estado de bienestar. Valencia: Institución Alfonso el Magnánimo.

ESPING ANDERSEN, G. (2000). Los fundamentos sociales de las economías postindustriales. Barcelona: Ariel.

ESPING ANDERSEN, G. (2006). Equal Opportunities in an Increasingly Hostile World.

ESPING ANDERSEN, G., \& MYLES, J. (2007). The Welfare State and Redistribution.

FERRARI RECALDE, O. G. (2008). Transmisión intergeneracional de la pobreza en Uruguay. Barcelona: Universidad Autónoma de Barcelona.

FILGUEIRA, C. (2000). La actualidad de viejas temáticas: sobre los estudios de clase, estratificación y movilidad social en América Latina. Santiago de Chile: CEPAL.

GOLDTHORPE, J., \& JACKSON, M. (n.d.). www.equalsoc.org. Obtenido el 13 de Marzo, 2012, de: http://www.equalsoc.org/uploaded_files/regular/goldthorpe_ jackson.pdf

GOLOVANEVSKY, L. (17 de Abril de 2007). Vulnerabilidad y transmición intergeneracional de la pobreza. Un abordaje cuantitativo para Argentina en el siglo XXI. La Plata: Tesis de doctorado. Obtenido el 05 de Abril, 2012, de: http://www.econ.uba.ar/www/servicios/Biblioteca/bibliotecadigital/bd/tesis_doc/gol ovanevsky.pdf

GUADALUPE, C. (2002). Indicadores de cobertura, eficiencia y flujo escolar: necesidades de política, problemas metodológicos y una propuesta. Organización de las Naciones Unidas para la Educación, la Ciencia y la Cultura. Santiago de Chile: UNESCO -.

INDEC. (2003). Acerca del método utilizado para la medición de La pobreza en Argentina. Dirección Nacional de Encuestas de Hogares del INDEC. Capital Federal: INDEC.

INFANTE, M. I. (2000). Alfabetismo funcional en siete países de América Latina. Santiago de Chile: UNESCO.

Jiménez, M. (2011). Un Análisis Empírico de las No Linealidades en la Movilidad Intergeneracional del Ingreso. El caso de la Argentina. Documentos de Trabajo(114).

LÓPEZ, N. (2002). Alcances y característicasde la repitencia, la sobreedad y el abandono en laArgentina. En OEA, Estrategias sistémicas de atención a la deserción, la repitencia yla sobreedad en escuelas de contextos desfavorecidos.Un balance de los años '90 en la Argentina. Buenos Aires: IIPE - UNESCO / OEA . 
MACHINEA, J. L. (2007). El financiamiento solidario de la protección social: condición indispensable para un pacto de cohesión social en América Latina. En A. Sojo, \& A. (. Uthoff, Cohesión social en América Latina y el Caribe: una revisión perentoria de algunas de sus dimensiones (págs. 23-34). Santiago de Chile: CEPAL.

MARTÍNEZ FRANZONI, J. (2005a). Regímenes de Bienestar en América Latina. ¿Cuáles y cómo son? Documento de Avances de Investigación.

MARTÍNEZ FRANZONI, J. (2005b). Regímenes de bienestar en América Latina: consideraciones generales e itinerarios regionales. Revista Centroamericana de Ciencias Sociales, 2(2), 41-77.

MÉNDEZ, M. L., \& GAYO, M. (2007). El perfil de un debate: movilidad y meritocracia. Contribución al estudio de las sociedades latinoamericanas. En F. Rolando, Estratificación y movilidad social en América Latina: transformaciones estructurales de un cuarto de siglo (págs. 121-161). Santiago de Chile: LOM Ediciones.

MORENO MÍNGUEZ, A. (Diciembre de 2008). Contextos explicativos de la reducida fecundidad y el reducido empleo femenino en España en el marco comparado europeo. Gizarte zerbitzuetarako aldizkaria(44), 65-76.

OBSERVATORIO SOCIAL. (2001). Oportunidades ocupacionales y distribución del ingreso. Ciudad Autonoma de BUenos Aires: Observatorio Social.

PAES DE BARROS, R., FERREIRA, F., MOLINAS VEGA, J., \& SAAVEDRA CHANDUVI, J. (2008). Midiendo la Desigualdad de Oportunidades en América Latina y el Caribe. Washington, EE.UU: Banco Mundial.

PARELLA RUBIO, S. (2000). Las políticas familiares. En J. Adelantado Gimeno, Cambios en el estado del bienestar: políticas sociales y desigualdades de España (págs. 415-442). Barcelona, España: Icaria : Universitat Autònoma de Barcelona, Servei de Publicacions.

SIEMPRO. (2001). El aislamiento social de los pobres urbanos: reflexiones sobre su naturaleza, determinantes y consecuencias. Buenos Aires: SIEMPRO.

TORCHE, F., \& WORMALD, G. (Octubre de 2004). Estratificación y movilidad social en Chile: entre la adscripción y el logro. Santiago de Chile, Chile: CEPAL.

TORRADO, S. (1994). Vivir apurado para morirse joven. Reflexiones sobre la transferencia intergeneracional de la pobreza. Revista Sociedad(7).

VELEZ, E., SCHIEFELBEIN, E., \& VALENZUELA, J. (1997). Factores que afectan el rendimiento académico en la educación primaria. Recuperado el Febrero de 2012, de OEI: http://www.mined.gob.ni/pdf2005/pdf2004/Factores\%20Asociados \%20Nicaragua\%202004.pdf 


\section{Correspondencia de los autores:}

María Sol TORRES MINOLDO

Facultad de Derecho

Universidad de Granada, España.

Calle Puente Espinosa $\mathrm{N}^{\circ} 20\left(2^{\circ} \mathrm{D}\right)$,

Granada 18009, España

razmujin@gmail.com

Marcos Javier ANDRADA

UNLaR - CIECS - CONICET - UNC.

Calle: Buenos Aires $330\left(11^{\circ} \mathrm{C}\right)$,

Córdoba, Argentina

licmarcosandrada@yahoo.com.ar 